\title{
Erratum to: Characterization of iodinated disinfection by-products in chlorinated and chloraminated waters using Orbitrap based gas chromatography-mass spectrometry
}

\author{
Cristina Postigo $^{1}$ (D) $\cdot$ Cristian I. Cojocariu $^{2} \cdot$ Susan D. Richardson ${ }^{3} \cdot$ Paul J. Silcock $^{2}$ • \\ Damia Barcelo ${ }^{1,4}$
}

Received: 12 July 2016 / Accepted: 12 July 2016/Published online: 8 August 2016

(C) Springer-Verlag Berlin Heidelberg 2016

\section{Erratum to: Anal Bioanal Chem}

DOI: 10.1007/s00216-016-9435-x

In the original version of this article, one of the chromatographic peaks found in NL NOM extracts was identified as iodoethene. However, the molecular structure proposed was not correct. Following Professor Albert T. Lebedev's suggestion, this peak may correspond to ethyl $\beta$-iodopropionate $\left(\mathrm{C}_{5} \mathrm{IO}_{2} \mathrm{H}_{9}\right)$. The authors completely agree with this suggestion, based on the following facts:

- The retention time for iodoethene should be shorter than that observed for ethyl iodoacetate $\left(t_{R}=8.07\right)$.

The online version of the original article can be found at http://dx.doi. org/10.1007/s00216-016-9435-x.

Cristina Postigo

cprqam@cid.csic.es

1 Department of Environmental Chemistry, Institute of Environmental Assessment and Water Research, (IDAEA-CSIC) Water and Soil Quality Research Group, Jordi Girona 18-26, Barcelona 08034, Spain

2 Thermo Fisher Scientific, Tudor Road, Manor Park, Runcorn, Cheshire WA7 1TA, UK

3 Department of Chemistry and Biochemistry, University of South Carolina, Columbia, SC, USA

4 Catalan Institute for Water Research (ICRA), Parc Científic i Tecnològic de la Universitat de Girona, Edifici H2O, Emili Grahit 101, 17003 Girona, Spain
On the other hand, ethyl $\beta$-iodopropionate should be retained in the column longer as compared to ethyl iodoacetate.

- The other ions in the mass spectrum, i.e., $87.04409\left(\mathrm{C}_{4} \mathrm{H}_{7} \mathrm{O}_{2}, 0.4 \mathrm{ppm}\right), 154.93519\left(\mathrm{C}_{2} \mathrm{H}_{4} \mathrm{I}\right.$, $-0.2 \mathrm{ppm})$, and $140.91956\left(\mathrm{CH}_{2} \mathrm{I}, 0.04 \mathrm{ppm}\right)$ also support the structural assignment as ethyl $\beta$ iodopropionate.

The authors would like to highlight that this peak misidentification is attributed only to the manual interpretation of the HRMS data and not to the GC-Orbitrap MS instrument performance.

Figure $3 b$ has been also changed accordingly, and ethyl $\beta$ iodopropionate should read throughout the text instead of iodoethene.

In order to confirm that this compound was a disinfection byproduct and not formed during an extraction process based on ethyl acetate, an additional blank of the chloramination process with purified water spiked with $500 \mathrm{ppb}$ of bromide and $50 \mathrm{ppb}$ of iodide (as $\mathrm{KBr}$ and $\mathrm{KI}$, respectively) was performed. Both, ethyl iodoacetate and ethyl $\beta$-iodopropionate were found in this blank extract; however, the peak areas in chloraminated and chlorinated extracts were comparatively much higher than in the blank (10 times and 5 times, respectively). Therefore, it was concluded that these compounds also were generated during the disinfection process.

Acknowledgments Authors would like to thank Prof. Albert T. Lebedev from the Lomonosov Moscow State University (Moscow, Russia) for his kind comments and help with EI MS interpretation. 
(b)
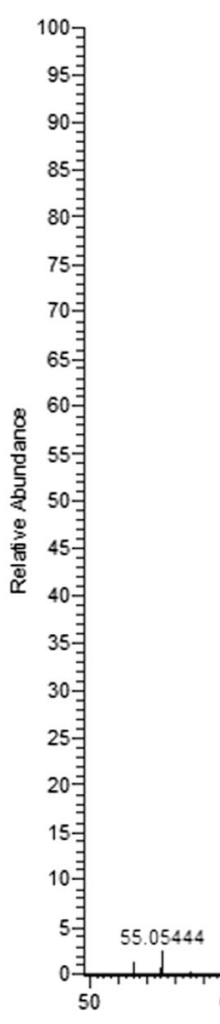<smiles>CCCC(CBr)(CC(=O)OCC)CC(C)(C)I</smiles>

53.92739

$\mathrm{C}_{2} \mathrm{H}_{3} \mathrm{I}$ $-0.01355 \mathrm{ppm}$ $\mathrm{C}_{4} \mathrm{H}_{7} \mathrm{O}_{2}$ $42543 \mathrm{ppm}$

Fig. 3 Fragment rationalization for mass spectrum of the peak appearing at $t_{R}=8.14 \mathrm{~min}$ in the chloraminated NL NOM extract 